\title{
Association of fluorescein anterior corneal mosaic and corneal K-structures by in vivo laser confocal microscopy in patients with keratoconus
}

This article was published in the following Dove Press journal:

Clinical Ophthalmology

26 July 2017

Number of times this article has been viewed

\section{Akira Kobayashi \\ Hideaki Yokogawa \\ Natsuko Mori \\ Toshinori Masaki \\ Kazuhisa Sugiyama}

Department of Ophthalmology, Graduate School of Medical Science, Kanazawa University, Kanazawa, Japan
Correspondence: Hideaki Yokogawa Department of Ophthalmology, Graduate School of Medical Science, Kanazawa University, I3-I Takara-machi, Kanazawa, Ishikawa-prefecture 920-864I, Japan Email hyoko@med.kanazawa-u.ac.jp
Objective: To report the in vivo laser confocal microscopy findings of corneas with keratoconus, with special attention to abnormality of Bowman's layer and sub-Bowman's fibrous structures (Kobayashi-structures [K-structures]).

Methods: Sixteen keratoconic eyes in 8 consecutive patients with keratoconus (4 males, 4 females, mean age, 41.1 years) were included in this study. Slit-lamp biomicroscopic photos were taken with or without fluorescein staining. The existence of anterior corneal mosaic (ACM) after eyelid rubbing under fluorescein staining was documented. In vivo laser confocal microscopic examinations were performed for all patients in both the central cone and the peripheral cornea to examine the existence of $\mathrm{K}$-structures.

Results: According to the Amsler-Krumeich scale, the eyes were graded as follows: stage 1 $(n=3)$, stage $2(n=1)$, stage $3(n=1)$, and stage $4(n=11)$. ACM was observed in 7 eyes $(61.1 \%)$ in the cone area and 16 eyes (100\%) in the peripheral cornea among all keratoconic eyes enrolled in this study. In addition, K-structures were observed in the 7 eyes $(61.1 \%)$ and 16 eyes $(100 \%)$ in the peripheral cornea among all keratoconic eyes. The presence of the K-structures was completely matched $(100 \%)$ with the presence of ACM in both the central cone and the peripheral cornea. In 11 eyes with stage 4 keratoconus, ACM and K-structure was absent in 9 eyes $(81.8 \%)$ in the cone area. On the contrary, in 5 eyes with mild-to-moderate keratoconus (grade 1 to 3), ACM and K-structure was present in all eyes (100\%) in the cone area. The absent ratio of ACM and K-structures in the cone area was significantly higher in stage 4 severe keratoconus compared to mild-to-moderate keratoconus (grade 1 to 3 ) (Fisher, $P=0.005$ )

Conclusion: The existence of ACM and K-structures in both the central cone and the peripheral cornea showed perfect accord in patients with keratoconus, indicating a strong association of ACM and $\mathrm{K}$-structures in patients with keratoconus. With the progress of the keratoconus, it seemed that ACM and K-structure progressively disappeared, suggesting Bowman's layer abnormalities due to keratoconus. Further study in larger groups of patients with keratoconus is required to fully understand the significance of $\mathrm{ACM} / \mathrm{K}$-structures in keratoconic eyes and their association with Bowman's layer.

Keywords: keratoconus, anterior corneal mosaic, K-structure, confocal microscopy

\section{Introduction}

Keratoconus is a noninflammatory ectatic thinning disorder of the cornea. ${ }^{1,2}$ The clinical manifestation of keratoconus is represented by a progressive astigmatism, which may lead to a significant reduction of visual acuity. Histopathology shows fragmentation and fibrillation of Bowman's layer and the epithelial basement membrane. ${ }^{1,2}$ Although the 
precise cause of keratoconus is still unclear, it is believed that genetics as well as environmental factors may have a role. ${ }^{1,2}$

Cornea-specific in vivo laser confocal microscopy (IVCM) (Heidelberg Retina Tomograph 2 Rostock Cornea Module, HRT2-RCM; Heidelberg Engineering GmbH, Heidelberg, Germany) has become available and has enabled more detailed in vivo observation of the corneal and conjunctival microstructure. ${ }^{3}$ Recently, analyses of the in vivo histology of all of the cell layers and nerves of patients with keratoconus were effectively performed with IVCM. $^{4-7}$

Previously, we used HRT2-RCM to demonstrate for the first time that there are fibrous structures just beneath Bowman's layer. ${ }^{8}$ These fibrous structures, designated "K-structures (Kobayashi-structures)," ${ }^{8}$ were 5-15 $\mu \mathrm{m}$ in diameter and appeared to consist of many filaments. ${ }^{8}$ We subsequently confirmed a strong association between K-structures and the presence/health of Bowman's layer. ${ }^{9-11}$ Therefore, we wondered whether there are pathological changes in K-structures in patients with keratoconus, a disease that might have Bowman's layer abnormalities.

In the current study, we report the in vivo laser confocal microscopy findings of corneas with keratoconus, with special attention to anterior corneal mosaic (ACM) and K-structures.

\section{Patients and methods}

This study was approved by the Ethical Committee of Kanazawa University Graduate School of Medical Science and followed the tenets of the Declaration of Helsinki.
Written informed consent was obtained from all patients and their parents if necessary. Sixteen keratoconic eyes in 8 consecutive patients with keratoconus ( 4 males, 4 females, age range from 19 to 57, mean age, 41.1 years) were included in this study (Table 1). The severity of keratoconus was classified according to the Amsler-Krumeich scale. Slit-lamp biomicroscopic photos were taken with or without fluorescein staining. The existence of ACM after eyelid rubbing under fluorescein staining was documented. In vivo laser confocal microscopic examinations were performed for all patients in both the central cone and the peripheral cornea.

\section{Results}

According to the Amsler-Krumeich scale, the eyes were graded as follows: stage $1(n=3)$, stage $2(n=1)$, stage $3(n=1)$, and stage $4(\mathrm{n}=11)$ (Table 1$)$. By slit-lamp biomicroscopy after eyelid rubbing under fluorescein staining, ACM was observed in 7 eyes $(61.1 \%)$ in the cone area and 16 eyes $(100 \%)$ in the peripheral cornea (black arrows, Figure 1C and F) among all keratoconic eyes enrolled in this study (Table 1). In addition, K-structures were observed in the 7 eyes (61.1\%) and 16 eyes (100\%) in the peripheral cornea among all keratoconic eyes. The presence of the K-structures was completely matched $(100 \%)$ with the presence of ACM in both the central cone and the peripheral cornea (Figures 1 and 2). In 11 eyes with stage 4 keratoconus, ACM and K-structure was absent in 9 eyes $(81.8 \%)$ in the cone area. On the contrary, in 5 eyes with mild-to-moderate keratoconus

Table I Association between ACM and K-structures at the cone and the peripheral cornea in patients with keratoconus

\begin{tabular}{|c|c|c|c|c|c|c|c|c|c|c|}
\hline \multirow[t]{2}{*}{ Case } & \multirow[t]{2}{*}{ Age } & \multirow[t]{2}{*}{ Sex } & \multirow{2}{*}{$\begin{array}{l}\text { Severity of } \\
\text { keratoconus }\end{array}$} & \multirow[t]{2}{*}{ Eye } & \multirow{2}{*}{$\begin{array}{l}\text { Max keratometry } \\
\text { and corneal } \\
\text { astigmatism (D) }\end{array}$} & \multirow{2}{*}{$\begin{array}{l}\text { Uncorrected and } \\
\text { best-corrected visual } \\
\text { acuity (decimal) }\end{array}$} & \multicolumn{2}{|l|}{ Cone } & \multicolumn{2}{|c|}{ Periphery } \\
\hline & & & & & & & $\mathrm{ACM}$ & $\overline{\text { K-structures }}$ & $\mathrm{ACM}$ & K-structures \\
\hline \multirow[t]{2}{*}{ I } & 57 & $\mathrm{~F}$ & Stage 4 & $\mathrm{R}$ & $71.9,-14.2$ & $0.01(0.02)$ & - & - & + & + \\
\hline & & & Stage 4 & $\mathrm{~L}$ & $73.9,-1.7$ & $0.02(0.03)$ & - & - & + & + \\
\hline \multirow[t]{2}{*}{2} & 40 & $\mathrm{~F}$ & Stage 4 & $\mathrm{R}$ & No data & $0.01(0.08)$ & + & + & + & + \\
\hline & & & Stage 4 & $\mathrm{~L}$ & No data & 0.01 (n.c.) & + & + & + & + \\
\hline \multirow[t]{2}{*}{3} & 34 & $M$ & Stage 4 & $\mathrm{R}$ & $78.9,-1.9$ & 0.03 (n.c.) & - & - & + & + \\
\hline & & & Stage I & $\mathrm{L}$ & $46.7,-4.0$ & I. 2 (n.c.) & + & + & + & + \\
\hline \multirow[t]{2}{*}{4} & 19 & $\mathrm{~F}$ & Stage 2 & $\mathrm{R}$ & $52.1,-5.2$ & $0.6(0.8)$ & + & + & + & + \\
\hline & & & Stage 4 & $\mathrm{~L}$ & $64.3,-2.3$ & $0.04(0.06)$ & - & - & + & + \\
\hline \multirow[t]{2}{*}{5} & 41 & $M$ & Stage I & $\mathrm{R}$ & $43.2,-3.9$ & I.2 (n.c.) & + & + & + & + \\
\hline & & & Stage 4 & $\mathrm{~L}$ & $76.4,-6.2$ & $0.02(0.03)$ & - & - & + & + \\
\hline \multirow[t]{2}{*}{6} & 47 & $\mathrm{~F}$ & Stage 4 & $\mathrm{R}$ & $59.7,-1.0$ & $0.1(1.2)$ & - & - & + & + \\
\hline & & & Stage 4 & $\mathrm{~L}$ & $60.5,-5.2$ & $0.1(0.8)$ & - & - & + & + \\
\hline \multirow[t]{2}{*}{7} & 45 & $M$ & Stage I & $\mathrm{R}$ & $43.3,-0.9$ & I.2 (n.c.) & + & + & + & + \\
\hline & & & Stage 3 & $\mathrm{~L}$ & $55.0,-5.6$ & $0.1(0.6)$ & + & + & + & + \\
\hline \multirow[t]{2}{*}{8} & 46 & $M$ & Stage 4 & $\mathrm{R}$ & $63.0,-0.2$ & $0.04(0.2)$ & - & - & + & + \\
\hline & & & Stage 4 & $\mathrm{~L}$ & $59.3,-5.8$ & $0.1(0.7)$ & - & - & + & + \\
\hline
\end{tabular}

Notes: +, presence; -, absence.

Abbreviations: ACM, anterior corneal mosaic; F, female; K-structure, Kobayashi-structure; L, left eye; M, male; n.c., non-correctable; R, right eye. 

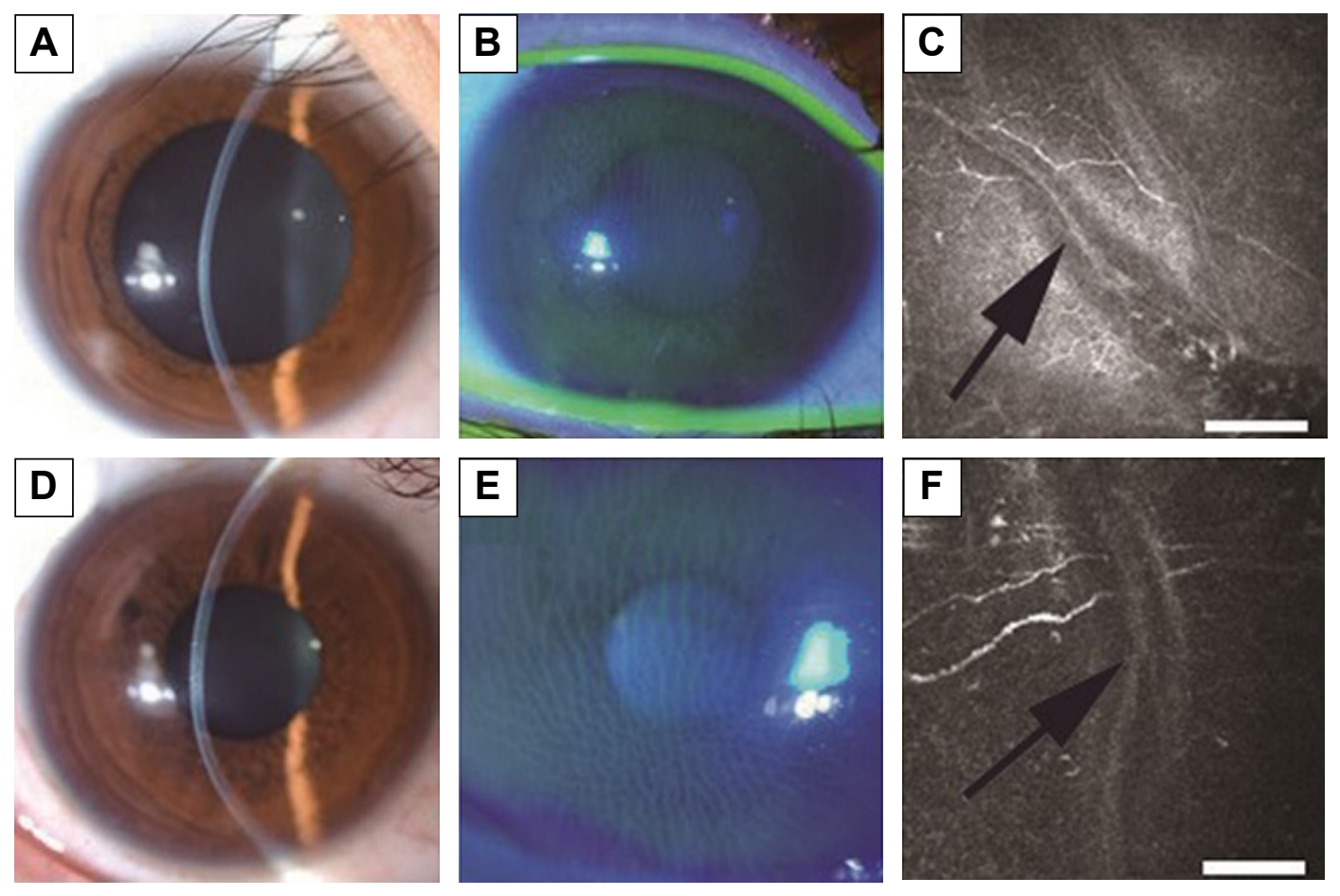

Figure I Representative cases of mild keratoconus with ACM.

Notes: Slit-lamp biomicroscopic photo with or without fluorescein staining and in vivo confocal microscopic image at the level of Bowman's layer. (A-C: case 4, right eye. D-F: case 5, right eye). (A) Slit-lamp biomicroscopic photo of the right eye in case 4. (B) After rubbing the right eye through the eyelid under fluorescein staining, corneal mosaic was apparent. (C) At the level of Bowman's layer, K-structures (sub-Bowman's fibrous structures) were apparent (bar =I $00 \mu \mathrm{m}$ ). (D) Slit-lamp biomicroscopic photo of the right eye in case 5. (E) After rubbing the right eye through the eyelid under fluorescein staining, corneal mosaic was apparent. (F) At the level of Bowman's layer, K-structures were apparent (bar $=100 \mu \mathrm{m})$.

Abbreviations: ACM, anterior corneal mosaic; K-structure, Kobayashi-structure.

(grade 1 to 3), ACM and K-structure were present in all eyes $(100 \%)$ in the cone area. The absent ratio of ACM and $\mathrm{K}$-structures in the cone area was significantly higher in stage 4 severe keratoconus compared to mild-to-moderate keratoconus (grade 1 to 3 ) (Fisher, $P=0.005$ ).

\section{Discussion}

We have previously reported that there are numerous fibrous structures just beneath Bowman's layer, and we designated them as "K-structures." Although the frequency of $\mathrm{K}$-structures has not investigated in a large control population, they were present in all healthy volunteers (100\%) in our previous study ( $\mathrm{n}=36$ eyes). Based on previous light/electron microscopic observations ${ }^{12-14}$ and two-photon-generated second-harmonic signal observations, ${ }^{15}$ we speculated that K-structures may correspond with anterior collagen fiber bundles running at the posterior surface of Bowman's layer. Subsequently, we reported that the overall distribution of $\mathrm{K}$-structures in normal human corneas by in vivo laser confocal microscopy formed a net-like mosaic pattern and corresponded quite well with the fluorescein ACM pattern observed with slit-lamp biomicroscopy. ${ }^{9}$ These results support the hypothesis that the K-structures are the anterior collagen fiber bundles running at the posterior surface of Bowman's layer, and thus are the structural basis for ACM formation. Therefore, we concluded that the presence of K-structures as observed by in vivo laser confocal microscopy and the presence of fluorescein ACM as observed by slit-lamp biomicroscopy can be an indicator of the health of Bowman's layer. Furthermore, we have reported that both K-structures and ACM disappeared only after epipolis-laser-assisted in situ keratomileusis (epi-LASIK), in which Bowman's layer is destroyed, but not after laser-assisted in situ keratomileusis (LASIK) in which Bowman's layer is preserved. ${ }^{10}$ Additionally, we have reported the absence of ACM and K-structures in patients with osteogenesis imperfecta, ${ }^{11}$ a disease in which Bowman's layer is believed to be completely missing, again confirming the strong association of $\mathrm{ACM} / \mathrm{K}$-structures and Bowman's layer.

In the current study, we investigated for the first time the association of ACM and K-structures by in vivo laser confocal microscopy in patients with keratoconus. As a result, the existence of ACM and K-structures in both the central cone and the peripheral cornea showed perfect accord in patients 

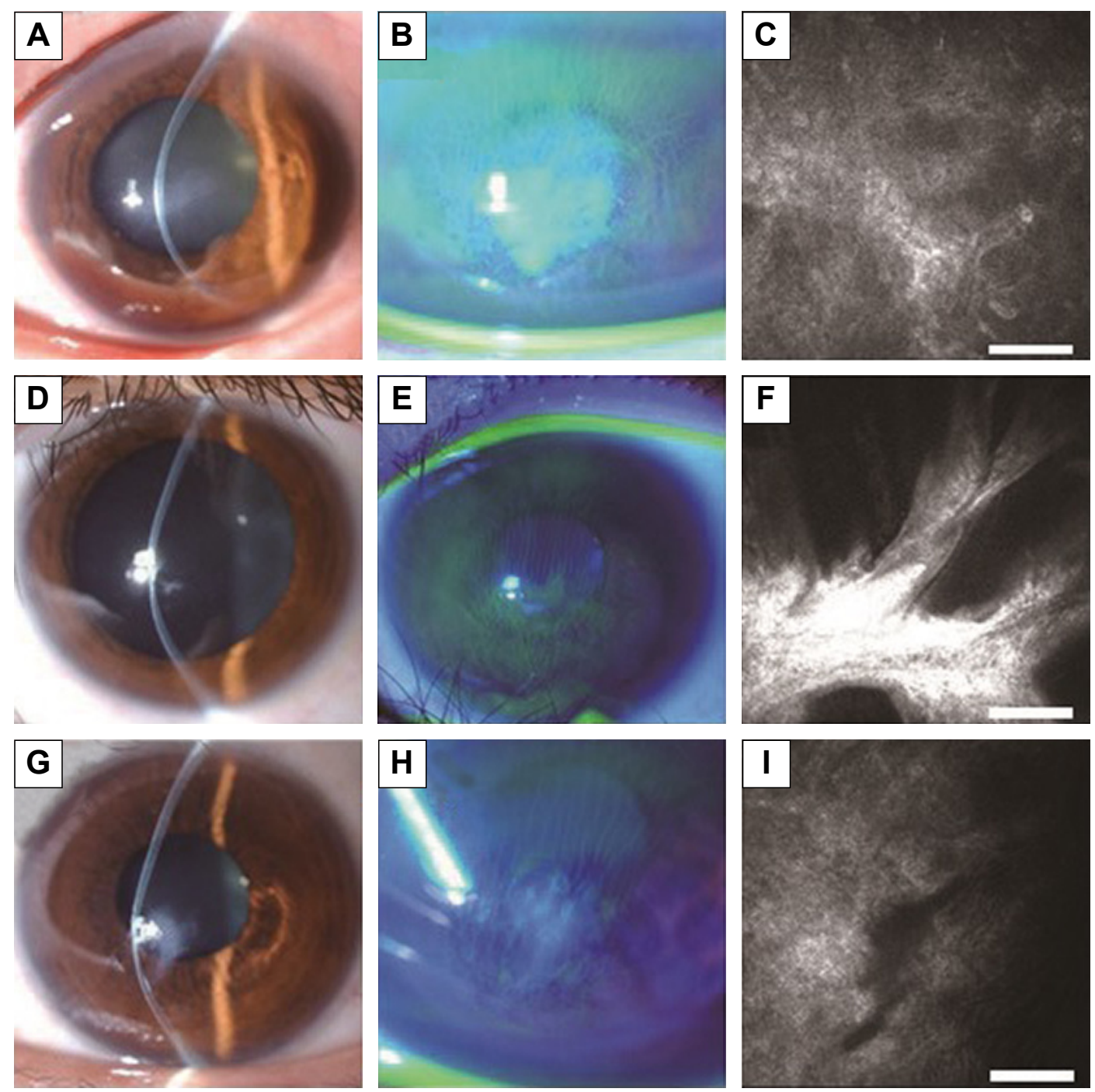

Figure 2 Representative cases of severe keratoconus without ACM.

Notes: Slit-lamp biomicroscopic photo with or without fluorescein staining and in vivo confocal microscopic image at the level of Bowman's layer. (A-C: case I, left eye. D-F: case 4, left eye. G-I: case 5, left eye.). (A) Slit-lamp biomicroscopic photo of the left eye in case I. (B) After rubbing the right eye through the eyelid under fluorescein staining, corneal mosaic was not apparent in the central cone area. (C) At the level of Bowman's layer, K-structures were absent in the central cone area (bar $=100 \mu \mathrm{m}$ ). (D) Slit-lamp biomicroscopic photo of the left eye in case 4. (E) After rubbing the right eye through the eyelid under fluorescein staining, corneal mosaic was not apparent in the central cone area. (F) At the level of Bowman's layer, K-structures were absent in the central cone area (bar $=100 \mu \mathrm{m}$ ). (G) Slit-lamp biomicroscopic photo of the left eye in case 5. (H) After rubbing the right eye through the eyelid under fluorescein staining, corneal mosaic was not apparent in the central cone area. (I) At the level of Bowman's layer, K-structures were absent in the central cone area $($ bar $=100 \mu \mathrm{m})$. Abbreviations: ACM, anterior corneal mosaic; K-structure, Kobayashi-structure.

with keratoconus, again confirming a strong association of ACM and K-structures, even in patients with keratoconus. All keratoconic eyes in this study showed both ACM and K-structures in the peripheral cornea, suggesting that Bowman's layer is relatively intact in the peripheral cornea. The absent ratio of ACM and K-structures in the cone area was significantly higher in stage 4 severe keratoconus compared to mild-to-moderate keratoconus (grade 1 to 3) (Fisher, $P=0.005)$. These results indicate that ACM and K-structure progressively disappeared with the progress of the keratoconus, suggesting Bowman's layer abnormalities. It should be noted that only case 2 presented K-structures and ACM in stage 4 keratoconus. Although the precise reason is unknown, we surmise that the scarred cone located relatively lower in case 2 and confocal microscopy might observe unaffected central cornea. We should also pay attention to the point that epithelial haze or fibrosis may prevent visualization of $\mathrm{K}$-structure by confocal microscopy and correct assessment of the presence/absence of K-structures, and so they might act as confounder.

Recently, Bitirgen et $\mathrm{al}^{7}$ reported the results of an in vivo confocal microscopic analysis in a large number of patients with keratoconus ( $n=78$ ) with the same device (HRT2-RCM). They reported significant microstructural abnormalities in all 
corneal layers quantitatively; these included enlarged basal epithelial cells, structural changes in subbasal and stromal nerve fibers, abnormal stromal keratocyte/keratocyte nuclei, and pleomorphism/enlargement of endothelial cells. They concluded that HRT2-RCM is a rapid noninvasive means to quantify all layers of the central cornea in keratoconic patients. However, they did not mention the structural changes of Bowman's layer or K-structures, as shown in this study.

\section{Conclusion}

In conclusion, the existence of ACM and K-structures in both the central cone and the peripheral cornea showed perfect accord in patients with keratoconus, indicating a strong association of ACM and K-structures in patients with keratoconus. With the progress of the keratoconus, it seemed that ACM and K-structure progressively disappeared, suggesting Bowman's layer abnormalities due to keratoconus. However, the drawback and limitation of this study are the small sample size and loss of histology/electron microscopy data. Further study in larger groups of patients with keratoconus is required to fully understand the significance of $\mathrm{ACM} / \mathrm{K}$-structures in keratoconic eyes and their association with Bowman's layer.

\section{Disclosure}

None of the authors has any proprietary interest in any product mentioned in this article.

The corresponding investigator (HY) has full access to all data in the study and takes responsibility for the integrity of the data and the accuracy of the data analysis. The authors report no conflicts of interest in this work.

\section{References}

1. Arffa RC. Dystrophies of the epithelium, Bowman's layer and stroma. Grayson's Disease of the Cornea. 4th ed. St Louis, MO: Mosby Inc; 1997: 413-463.
2. Romero-Jimenez M, Santodomingo-Rubido J, Wolffsohn JS. Keratoconus: a review. Cont Lens Anterior Eye. 2010;33(4):157-166.

3. Zhivov A, Stachs O, Kraak R, Stave J, Guthoff RF. In vivo confocal microscopy of the ocular surface. Ocul Surf. 2006;4:81-93. Review.

4. Patel DV, Ku JY, Johnson R, McGhee CN. Laser scanning in vivo confocal microscopy and quantitative aesthesiometry reveal decreased corneal innervation and sensation in keratoconus. Eye (Lond). 2009; 23(3):586-592.

5. Ku JY, Niederer RL, Patel DV, Sherwin T, McGhee CN. Laser scanning in vivo confocal analysis of keratocyte density in keratoconus. Ophthalmology. 2008;115(5):845-850.

6. Patel DV, McGhee CN. Mapping the corneal sub-basal nerve plexus in keratoconus by in vivo laser scanning confocal microscopy. Invest Ophthalmol Vis Sci. 2006;47(4):1348-1351.

7. Bitirgen G, Ozkagnici A, Bozkurt B, Malik RA. In vivo corneal confocal microscopic analysis in patients with keratoconus. Int J Ophthalmol. 2015;8(3):534-539.

8. Kobayashi A, Yokogawa H, Sugiyama K. In vivo laser confocal microscopy of Bowman's layer of the cornea. Ophthalmology. 2006;113: 2203-2208.

9. Yokogawa H, Kobayashi A, Sugiyama K. Mapping of normal corneal K-structures by in vivo laser confocal microscopy. Cornea. 2008;27: 879-883.

10. Yokogawa H, Kobayashi A, Tagawa K, Sugiyama K. In vivo laser confocal microscopic analysis of corneal K-structures after keratorefractive surgery (LASIK and epi-LASIK). Ophthalmic Surg Lasers Imaging. 2010;41:494-498.

11. Kobayashi A, Higashide T, Yokogawa H, Yamazaki N, Masaki T, Sugiyama K. In vivo laser confocal microscopy findings of a cornea with osteogenesis imperfecta. Clin Ophthalmol. 2014;8:429-433.

12. Hogan MJ, Alvarado JA, Weddell E. Histology of the Human Eye. Philadelphia, PA: WB Saunders; 1971:55-111.

13. Komai $Y$, Ushiki $T$. The three-dimensional organization of collagen fibrils in human cornea and sclera. Invest Ophthalmol Vis Sci. 1991; 32:2244-2258.

14. Smolek MK, Klyce SD. Cornea. In: Tasman W, Jaeger E, editors. Duane's Foundation of Clinical Ophthalmology. Volume 1. Ocular Anatomy, Embryology, and Teratology [book on CD-ROM]. Philadelphia, PA: Lippincott Williams \& Wilkins; 2006.

15. Morishige N, Petroll WM, Nishida T, Kenney MC, Jester JV. Noninvasive corneal stromal collagen imaging using two-photongenerated second-harmonic signals. J Cataract Refract Surg. 2006;32: 1784-1791.
Clinical Ophthalmology

\section{Publish your work in this journal}

Clinical Ophthalmology is an international, peer-reviewed journal covering all subspecialties within ophthalmology. Key topics include: Optometry; Visual science; Pharmacology and drug therapy in eye diseases; Basic Sciences; Primary and Secondary eye care; Patient Safety and Quality of Care Improvements. This journal is indexed on Submit your manuscript here: http://www.dovepress.com/clinical-ophthalmology-journal

\section{Dovepress}

PubMed Central and CAS, and is the official journal of The Society of Clinical Ophthalmology (SCO). The manuscript management system is completely online and includes a very quick and fair peer-review system, which is all easy to use. Visit http://www.dovepress.com/ testimonials.php to read real quotes from published authors. 